\title{
Regulation of BGC-823 cell sensitivity to adriamycin via miRNA-135a-5p
}

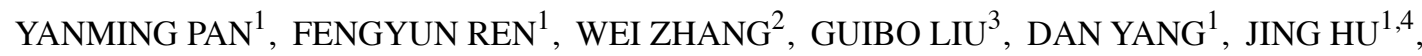 \\ KEJIAN FENG ${ }^{3}$ and YUKUAN FENG ${ }^{1,4,5}$
}

\author{
${ }^{1}$ Key Laboratory of Tumor Prevention and Treatment (Heilongjiang Higher Education Institutions), \\ Mudanjiang Medical University; ${ }^{2}$ Department of Respiration, Hongqi Hospital of Mudanjiang Medical University; \\ ${ }^{3}$ School of Basic Medical Science, Mudanjiang Medical University, Mudanjiang 157011; ${ }^{4}$ School of Basic \\ Medical Science, Harbin Medical University, Harbin 150086, P.R. China; ${ }^{5}$ Department of Cellular and \\ Molecular Medicine, University of California, San Diego, CA 92093, USA
}

Received August 7, 2014; Accepted September 26, 2014

DOI: 10.3892/or.2014.3546

\begin{abstract}
MicroRNAs (miRNAs) play an important role in the genesis and development of gastric cancer. In the present study, we determined whether miRNA-135a-5p expression was increased in gastric cancer compared with adjacent non-tumor tissues using 20 pairs of gastric cancer and para-carcinoma tissue samples which were assessed via microarray and bioinformatics analysis, and western blotting. The protein content detection showed that miRNA-135a-5p expression was inversely correlated with AP- $2 \alpha$. Bioinformatics analysis revealed that AP- $2 \alpha$ contains a putative miRNA-135a-5p target, which was confirmed as a direct target using the 3'-UTR luciferase reporter system. Additionally, an increase and decrease of miRNA-135a-5p inhibited or impaired adriamycin-induced apoptosis in BGC-823 cells $(\mathrm{p}<0.05$, compared with the group without gene intervention), respectively. Luciferase reporter experiments confirmed that AP- $2 \alpha$ bound to the BCL- 2 promoter and affected its transcription. Therefore, miRNA-135a-5p increased BCL-2 via AP- $2 \alpha$ and consequently enhanced cell resistance to apoptosis. This newly identified miRNA-135a-5p-AP-2 $\alpha$-BCL-2 pathway provides insight for the treatment of gastric cancer and solution for insensitivity of gastric cancer to chemotherapy drugs.
\end{abstract}

\section{Introduction}

Gastric carcinoma remains the fourth most common tumor and the second most common cause of cancer death world-

Correspondence to: Dr Yukuan Feng, Key Laboratory of Tumor Prevention and Treatment, Mudanjiang Medical University, 3 Tongxiang Street, Mudanjiang 157011, P.R. China

E-mail: fengyukuan@163.com

Professor Kejian Feng, School of Basic Medical Science, Mudanjiang Medical University, 3 Tongxiang Street, Mudanjiang 157011,P.R. China E-mail: fkj88812003@163.com

Key words: gastric cancer, AP-2 $\alpha$, anti-apoptosis, miRNA135a-5p, BCL-2 wide (1). Despite gradual use of new therapeutic approaches, the mortality rate caused by gastric carcinoma remains high. Surgery is a mainstay for gastric cancer treatment, however, it presents a poor outcome since a majority of patients with gastric cancer are diagnosed at an advanced stage. Therefore, treatment depends mainly on chemotherapy . However, there are no normative chemotherapeutic schemes for gastric cancer. The 5-year survival rate of patients diagnosed at an advanced stage is $<30 \%$ and $70 \%$ for those diagnosed at an early stage (2). Therefore, it is important to identify the molecular mechanism underlying the genesis and development of gastric cancer and to identify reliable biomarkers and therapeutic targets, consequently extending the lifespan of patients.

Recent studies have revealed that the roles of microRNAs (miRNAs) in the genesis and development of cancer have been increasingly understood along with ongoing investigations (3-5). miRNAs are known to be involved in many human tumors: in patients with intestinal or pancreatic cancer, for instance, a high expression of miR-10b correlates significantly with invasion and poor prognosis $(6,7)$, and a high miR-21 level is positively correlated with later clinical stages of rectal carcinoma and poorly differentiated cells (8). miRNA182 and miRNA205 were found to be closely associated with the genesis and development of breast cancer $(9,10)$. Chao et al (11) found that abnormal miR-187 expression is involved in the genesis of ovarian cancer and its role varies in different stages. Evidence suggests that the abnormal expression of miRNAs contributes to resistance of various types of cancer to chemotherapy: miR-20a can affect the sensibility of SW620 and SW680, two colorectal adenocarcinoma cell lines, to chemotherapeutics and the effects have been shown to be associated with its target gene BNIP2 (12); miR-32 regulates cancer cell sensibility to chemotherapeutics via its target gene Bim (13); miR-200c and mir-451 play roles in drug resistance of MCF-7 breast cancer cells, the former negatively regulating MDR1, thus increasing chemosensitivity of MCF-7/ADR to epirubicin (14) and the latter increasing resistance of tumor cells to adriamycin-induced apoptosis through the negative regulation of its target gene $P$ - $g p$ (15). Findings of previous 
studies have shown that miRNA21, miR-130b, miR-650 and miR-150 are involved in the genesis and development of gastric carcinoma (16-20). Tumor-promoting miR-421 and miR-106a are potential tumor markers in the diagnosis of gastric cancer $(21,22)$. Moreover, miRNA-15b, miRNA16 and miRNA497 may affect the drug resistance of gastric carcinoma cells by regulating BCL-2 $(23,24)$. Results of the aforementioned studies suggest that miRNAs are closely associated with the genesis and development of gastric cancer and play important roles in the chemoresistance of gastric cancer.

The aim of the present study was to determine whether miRNA-135a-5p expression was increased in gastric cancer compared with adjacent non-tumor tissues. The microarray analysis revealed that miRNA135a-5p is one of the miRNAs that were significantly differentially expressed in human gastric cancer samples. We isolated 20 pairs of gastric cancer and para-carcinoma tissue samples and detected mature miRNA135a-5p levels and its potential target AP- $2 \alpha$, predicted by bioinformatics analysis. Using quantitative PCR (qPCR) and western blotting, respectively, possible correlations were investigated. Furthermore, we verified the prediction using a luciferase reporter gene system. Chemically synthetic RNA was used to alter miRNA135a-5p content in BGC-823 cells and its effects on AP- $2 \alpha$ and proliferative activity and resistance to adriamycin-induced apoptosis were investigated. As a transcription factor, AP-2 $\alpha$ plays a role in tumor inhibition (25-27), although its direct involvement in chemoresistance remains to be determined. miRNA-135a-5P may regulate a key gene associated with proliferation or apoptosis via AP- $2 \alpha$. A possible binding site of AP- $2 \alpha$ in BCL-2 promoter through bioinformatics analysis was identified and verified using a luciferase reporter gene system. Additionally, we attempted to elucidate the regulatory mechanism by exploring effects of miRNA135a$5 \mathrm{p}$ on $B C L-2$, a crucial gene in cell proliferation and apoptosis.

\section{Materials and methods}

Prediction of seed region of miRNA135a-5p in AP-2 $\alpha$ mRNA and the binding site of $A P-2 \alpha$ in BCL-2 promoter. TargetScan was used to predict the possible target (seed region) of miRNA135a-5p in mRNA sequence of AP-2 $\alpha$ (NM_003220.2) and TFSEARCH software was used to identify the potential binding site of AP- $2 \alpha$ in BCL-2 promoter.

Vector construction and RNA synthesis. AP- $2 \alpha$ human 3'-untranslated region (3'-UTR, $314 \mathrm{bp}$ ) was amplified from cDNA obtained through the reverse transcription of total RNA of 293 cells, using the primers: 5'-GCTCTAGATGTGGAG CCTAAGAGAACAGA-3' and 5'-GCTCTAGAAATTCGT GTATTTGTGTTC-3'. The amplification parameters used were: 32 cycles of denaturation at $95^{\circ} \mathrm{C}$ for $10 \mathrm{sec}$, annealing at $58^{\circ} \mathrm{C}$ for $30 \mathrm{sec}$ and extension at $72^{\circ} \mathrm{C}$ for $30 \mathrm{sec}$. The product was digested with $\mathrm{XbaI}$ and inserted into the pGL3-promoter vector (Promega, Madison, WI, USA). The seed region was mutated from 5'-AGCCATA-3' to 5'-ACAGACT-3' by point mutation. The resulting vectors were designated as pGL-WTAP- $2 \alpha$ and pGL-MT-AP- $2 \alpha$, respectively. Sequences of the constructed vector were verified by sequencing. Human genomic DNA was extracted from 293 cells and the BCL-2 promoter sequence (694 bp) was amplified using the primers:
5'-GCTCTAGACAGGAGGAGGAGAAAGGGT-3' and 5'-GCTCTAGAAAACAAATGCATAAGGCAACGATC-3'. The cycling parameters were 32 cycles of denaturation at $95^{\circ} \mathrm{C}$ for $10 \mathrm{sec}$, annealing at $60^{\circ} \mathrm{C}$ for $30 \mathrm{sec}$ and extension at $72^{\circ} \mathrm{C}$ for $45 \mathrm{sec}$. The PCR product was digested and inserted into PGL3-enhancer (Promega), a luciferase reporter vector. The binding site was mutated from 5'-ACCGGCGGGCC-3' to 5'-GCAGCGCGCCG-3'. The resulting luciferase vectors were designated as pGL3-WT-BCL-2 and pGL3-MT-BCL-2, respectively. Sequences of the constructed vector were verified by sequencing. Chemically synthesized miRNA135a-5pmimics, inhibitor and NC were obtained from Shanghai Sangon (Shanghai, China).

Cell culture and treatment and luciferase assay. A total of 293 cells at $\log$ phase were suspensed and seeded in 96-well plates. For groups of AP- $2 \alpha$ overexpression and silencing, Lv-AP-2 $\alpha$ (Santa Cruz Biotechnology, Inc., Santa Cruz, CA, USA) and Lv-shRNA-AP- $2 \alpha$ viruses were used for infection at a MOI of 5. Transfection was carried out $24 \mathrm{~h}$ later, using Lipofectamine 2000 according to the manufacturer's instructions. The cells were also transfected with $50 \mathrm{ng}$ pRL-TK (Promega) for luciferase reference. After $48 \mathrm{~h}$, the cells were collected and the relative luciferase activities were measured by the dual luciferase reporter assay system (Promega). BGC-823 cells (Cell bank of Chinese Academy of China, Shanghai, China) were suspended and seeded in 6-well plates at $1 \times 10^{5}$ cells/well and cultured for $24 \mathrm{~h}$. For AP- $2 \alpha$ overexpression and silencing, Lv-AP-2 $\alpha$ (Santa Cruz Biotechnology, Inc.) and Lv-shRNA-AP- $2 \alpha$ viruses were used for infection at a MOI of 10. The infection efficiency was estimated by fluorescence microscopy. The cells were reseeded and transfected with miRNA135a-5p-mimics, inhibitor or NC sequence and collected for BCL mRNA and protein measurement.

Detection of miRNA135a-5p and BCL-2 mRNA using real-time $P C R$. Twenty pairs of gastric cancer and adjacent tissue samples were obtained from the Department of Gastroenterology of the Changhai Hospital (Shanghai, China). Each sample $(\sim 50 \mathrm{mg})$ was rinsed with $1 \mathrm{ml}$ cooled DPBS and $1 \mathrm{ml}$ pre-cooled TRIzol was added before subjecting to RNA extraction. Total RNA ( $2 \mu \mathrm{g})$ was used for cDNA preparation using the M-MLV reverse transcription kit (Takara Bio, Dalian, China) and the specific primers used were: U6 snRNA (NM_001101.3), 5'-TACCTTGCGAAGTGCTTAAAC-3' and miRNA135a-5p, 5'-GTCGTATCCAGTGCGTGTCGTGGA GTCGGCAATTGCACTGGATACGACTCACA-3'. RNA contents were detected using PCR of fluorescent dye (Takara Bio) according to the manufacturer's instructions. The primers used for quantification of human U6 snRNA and miRNA-137 were: U6 snRNA, 5'-GTGCTCGCTTCGGCAGCACAT-3' and 5'-TACCTTGCGAAGTGCTTAAAC-3', producing a segment of $211 \mathrm{bp}$; and miRNA-137: 5'-GCCGGCGCCCGA GCTCTGGCTC-3' and 5'-TATGGCTTTTTATTCCTATG TGA-3', producing a segment of $214 \mathrm{bp}$. PCR systems were: Takara SYBR Premix Ex Tap $10 \mu 1$, forward and reverse primers $(20 \mu \mathrm{M}) 0.2 \mu \mathrm{l}$ each and cDNA $2 \mu \mathrm{l}$, followed by the addition of $\mathrm{dH}_{2} \mathrm{O}$ to $20 \mu \mathrm{l}$. Cycling parameters used were: 40 cycles of denaturation at $95^{\circ} \mathrm{C}$ for $10 \mathrm{sec}$, annealing at $60^{\circ} \mathrm{C}$ for $20 \mathrm{sec}$ and extension at $72^{\circ} \mathrm{C}$ for $20 \mathrm{sec}$. U6 snRNA was 

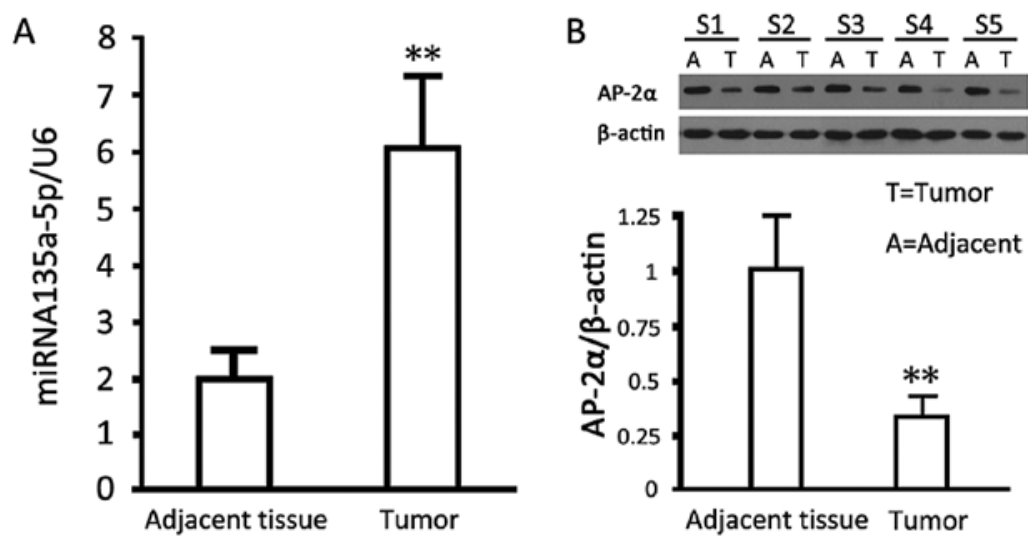

Figure 1. Analysis on contents of miRNA135a-5p and AP-2 $\alpha$ in gastric cancer and adjacent tissues. Twenty pairs of gastric cancer and adjacent tissues were collected and subjected to quantitative analysis of (A) miRNA135a-5p levels and western blotting (B) of AP-2 $\alpha$. The data are presented as the means \pm SD $(\mathrm{n}=3){ }^{* *} \mathrm{P}<0.01$.

used as a reference to normalize miRNA-137 levels using the $2^{-\Delta \Delta C T}$ method. Each RNA sample was run in triplicate. Total RNA was isolated from BGC-823 cells infected with Lv-AP- $2 \alpha$ or Lv-shRNA-AP- $2 \alpha$ virus and then transfected with miRNA135a-5p-mimics, inhibitor or NC sequence $48 \mathrm{~h}$ after transfection and reverse transcribed into cDNA. BCL-2 mRNA contents were subsequently detected using the primers: 5'-TGCACCTGACGCCCTTCACCG-3' and 5'-TTATCCTGG ATCCAGGTGTGC-3'.

Western blotting. The tissue samples mentioned above were also used for AP- $2 \alpha$ protein measurement. Each sample $(\sim 100 \mathrm{mg})$ was rinsed with $1 \mathrm{ml}$ cooled dPBS and added with $1 \mathrm{ml}$ tissue lysis buffer $(50 \mathrm{mM} \mathrm{pH} 8.0$ Tris, $1 \mathrm{mg} / \mathrm{ml}$ leupeptin, $150 \mathrm{mM} \mathrm{NaCl}, 0.5 \%$ Nonidet P-40, 5 mM EDTA, $100 \mathrm{mM}$ phenylmethylsulfonyl fluoride, $1 \mathrm{M}$ dithiolthretol and $1 \mathrm{mg} / \mathrm{ml}$ aprotinin) for protein extraction. Protein concentrations were detected by BCA assay. Protein samples $(11 \mu \mathrm{l})$ were separated by SDS-PAGE and transferred to PVDF membranes. Blots were blocked in TBST containing 5\% non-fat milk at room temperature for $2 \mathrm{~h}$ and incubated with the primary antibodies against AP- $2 \alpha$ and $\beta$-actin (AP- $2 \alpha$ 1:300 and $\beta$-actin 1:800; both from Santa Cruz) at $4^{\circ} \mathrm{C}$ overnight. Bands were detected with ECL chemiluminescence substrates (Pierce Biotechnology, Inc. Rockford, IL, USA) and the optical densities were analyzed with the image processing software. The relative content of AP- $2 \alpha$ was calculated as optical density of AP- $2 \alpha$ band/optical density of $\beta$-actin band. Western blotting was also used to detect AP- $2 \alpha$ and BCL- 2 changes in cells infected with or without Lv-AP- $2 \alpha$ or Lv-shRNA-AP-2 $\alpha$ viruses and then transfected with miRNA135a-5p-mimics, inhibitor or NC sequence. The primary antibody against BCL-2 was diluted at 1:500.

Cell proliferation and apoptosis assays. BGC-323 cells transfected with miRNA135a-5p-mimics, inhibitor or NC sequence were reseeded into 96 -well plates at $5 \times 10^{4}$ cells/well after 24-h transfection. Adriamycin (Sigma-Aldrich, St. Louis, MO, USA) was then added to a final concentration of 1,5 or $25 \mu \mathrm{g} / \mathrm{ml}$. CCK-8 solution (10 $\mu \mathrm{l}$; Dojindo, Osaka, Japan) was added into each well at different time points. The absorbance at $450 \mathrm{~m}$ was determined after an additional 4-h incubation. The
Annexin V-FITC Apoptosis Detection kit II (BD Biosciences, Pharmingen, CA, USA) was used for apoptosis analysis. Briefly, the cells were collected, washed with dPBS and suspended in $500 \mu \mathrm{l}$ binding buffer and stained with $5 \mu \mathrm{l}$ Annexin V-FITC in the dark for $10 \mathrm{~min}$ and then stained with $5 \mu \mathrm{l}$ propidium iodide for 5 min. Flow cytometry (FACSCalibur; BD Biosciences) was conducted using FL1 channel for Annexin V-FITC and FL2 channel for PI (FACSCalibur) at an excitation wavelength of $488 \mathrm{~nm}$.

Statistical analysis. SPSS13.0 was used for statistical analysis. Values are presented as the means \pm standard deviation (SD). Factorial analysis was employed for the inter- and intra-group comparison. $\mathrm{P}<0.05$ was considered significant.

\section{Results}

Analysis on the relative levels of miRNA135a-5p and AP-2 $\alpha$ in gastric cancer and para-carcinoma tissues. Quantitative results showed that miRNA135a-5p levels in gastric cancer tissues were significantly higher than those in para-carcinoma tissues $(\mathrm{P}<0.05)$. In comparison with those for para-carcinoma tissues, western blotting results showed that AP-2 $\alpha$ expressed in cancer tissues was significantly decreased ( $p<0.05)$ (Fig. 1).

Verification of interaction between miRNA135a-5p and $A P-2 \alpha$. The analysis of TargetScan showed that there is a possible binding site (seed region), 5'-AGCCAUA-3', in 3'-UTR of the AP-2 $\alpha$ gene, between bases 478-484 (Fig. 2A). 3'-UTR of AP-2 $\alpha$ was cloned into the pGL-3 luciferase reporter vector for verification. Luciferase activity detection showed that the miRNA135a-5p-mimic significantly inhibited intercellular luciferase activity $(\mathrm{P}<0.05$, compared with the group transfected with the luciferase expression vector alone) and miRNA135a-5p-inhibitor slightly increased the luciferase activity without reaching statistical significance. However, the two vectors did not directly affect the luciferase activity in cells transfected with the luciferase expression vector carrying a mutated binding site; in comparison with the group transfected the luciferase expression vector alone, the cells transfected with miRNA135a-5p-NC showed a similar lucif- 
A

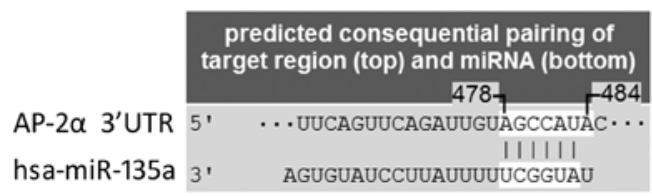

B

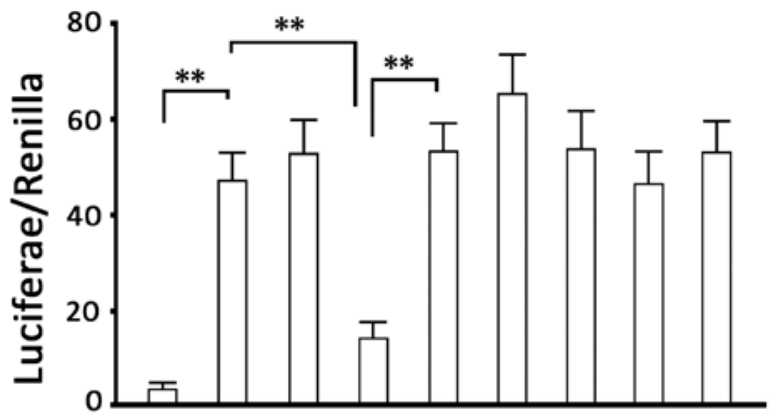

$\begin{array}{llllllllll}\text { pGL3-WT-AP-2 } \alpha & - & + & - & + & - & + & - & + & - \\ \text { pGL3-MT-AP-2 } \alpha & - & - & + & - & + & - & + & - & + \\ \text { pGL-TK } & + & + & + & + & + & + & + & + & + \\ \text { mimics } & - & - & - & + & + & - & - & - & - \\ \text { inhibitor } & - & - & - & - & - & + & + & - & - \\ \text { NC } & - & - & - & - & - & - & - & + & +\end{array}$

Figure 2. Verification of interaction between miRNA135a-5p and AP-2 $\alpha$. (A) Predicted binding site of miRNA135a-5p in 3'-UTR of the AP-2 $\alpha$ gene; (B) 293 cells were transfected with pGL3-WT-AP-2 $\alpha$ or pGL3-MT-AP- $\alpha$ and with or without miRNA135a-5p-mimic or miRNA135a-5p-inhibitor. The histogram indicates relative firefly luciferase activities in the different transfected groups. Error bars are the standard deviation and were obtained from at least three independent experiments. ${ }^{* *} \mathrm{P}<0.01$.

erase activity, indicating that RNA transfection had no effect on luciferase activity (Fig. 2B). These results suggested that the binding site of has-miRNA135a-5p in AP-2 $\alpha$ is in line with the predicted sequence.

Effects of miRNA135a-5p intervention on proliferation and drug resistance in BGC-823 cells. Analysis on relative levels of miRNA135a-5p and AP-2 $\alpha$ in transfected BGC-823 cells was detected. The results of miRNA135a-5p showed that miRNA135a-5p-mimics significantly increased miRNA135a$5 p$ content $(\mathrm{P}<0.05$, compared with the non-transfected group) although the inhibitor significantly decreased miRNA135a-5p content $(\mathrm{P}<0.05$, compared with the non-transfected group), and the transfection control group showed no difference as compared to the non-transfected group (Fig. 3A). Protein content detection showed that miRNA135a-5p-mimic significantly decreased AP- $2 \alpha$ levels $(\mathrm{P}<0.05$, compared with the untransfected group), whereas the miRNA135a-5p-inhibitor significantly increased AP- $2 \alpha$ expression $(\mathrm{P}<0.05$, compared with the non-transfected group). No difference was observed between the transfection control and non-transfected groups (Fig. 3B).

To investigate the effects of changes in miRNA135a-5p on the proliferation of BGC-823 cells, CCK-8 was used to analyze the proliferation of BGC-823 cells transfected with miRNA135a-5p-mimics, inhibitor and NC $48 \mathrm{~h}$ after transfection. The data suggested that high miRNA135a-5p content promoted cell proliferation at the logarithmic phase, although no statistically significant difference was observed $(\mathrm{P}>0.05$, vs. the control group). The cell viabilities in the group treated with the transfection agent and the group transfected with $\mathrm{NC}$ sequence were not altered ( $\mathrm{P}>0.05$, vs. the control group) (Fig. 3C). The cells were treated with adriamycin at different concentrations. A cell viability assay was carried out $24 \mathrm{~h}$ later and the results showed that miRNA135a-5p-mimics increased the sensitivity of BGC-823 cells to adriamycin, $(\mathrm{P}<0.05$, compared with the non-transfected group) and miRNA135a$5 \mathrm{p}$-inhibitor decreased this sensitivity $(\mathrm{P}<0.05$, compared with the non-transfected group). The cell viability of the transfection control group was not different to the cells treated with adriamycin alone (Fig. 3D).

To investigate the effects of miRNA135a-5p changes on adriamycin-induced apoptosis in BGC-823 cells, adriamycin $(5 \mu \mathrm{g} / \mathrm{ml})$ was used to treat BGC-823 cells, $24 \mathrm{~h}$ after transfection and apoptosis was detected. Our results showed that miRNA135a-5p-mimic transfection increased the sensitivity to adriamycin and the apoptotic rate induced by adriamycin and miRNA135a-5p-inhibitor impaired adriamycin-induced apoptosis $(\mathrm{P}<0.05$, vs. the group without gene intervention) (Fig. 3E and F).

Verification of binding site of $A P-2 \alpha$ in $B C L-2$ promoter. The results of the TFSEARCH analysis revealed a possible binding site 5'-ACCGGCGGGCC-3' of AP- $2 \alpha$ in the BCL-2 promoter. Luciferase activity analysis showed that the overexpression of AP- $2 \alpha$ increased luciferase activity in the group transfected 
A

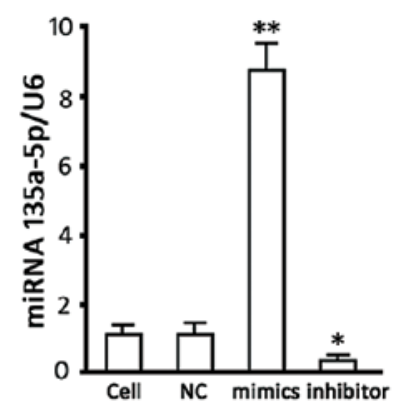

C

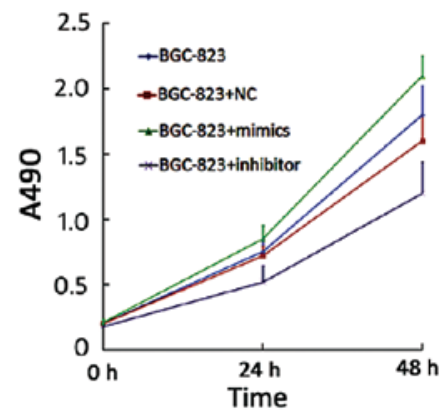

E
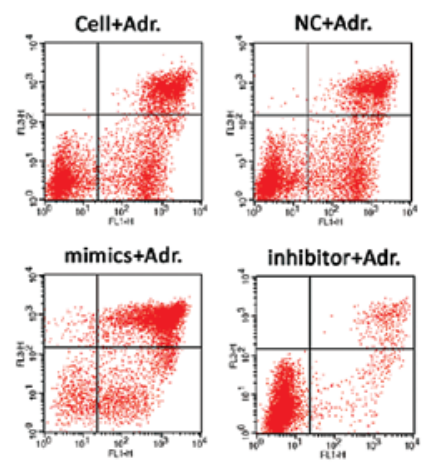

B
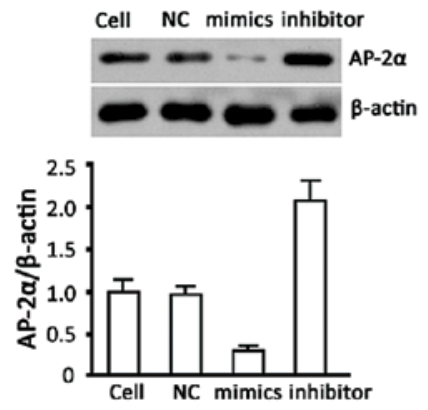

D

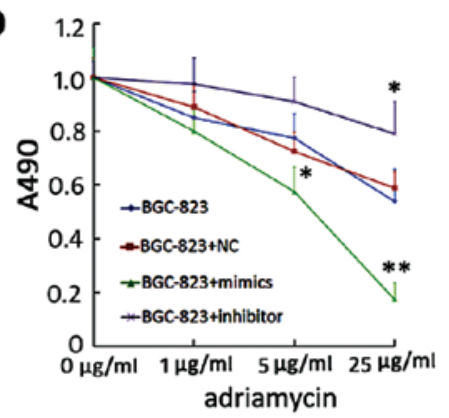

$\mathbf{F}$

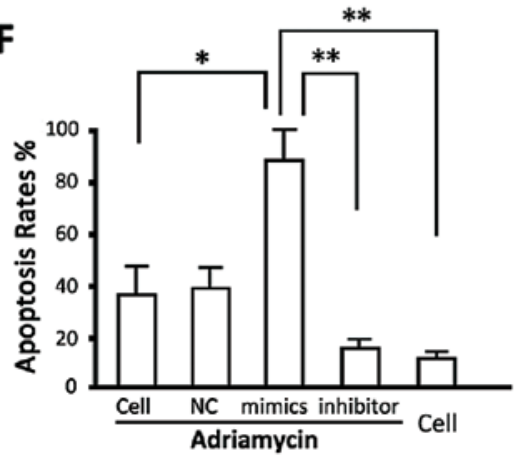

Figure 3. Association of miRNA135a-5p contents, protein levels, cell proliferation and apoptosis. BGC-823 cells were transfected by miRNA135a-5p-mimics, miRNA135a-5p-inhibitor or NC sequence and were subject to quantitative PCR detection for (A) miRNA135a-5p and western blotting of (B) AP-2 $\alpha 48 \mathrm{~h}$ after transfection, or were reseeded in 96-well plates $24 \mathrm{~h}$ after transfection and subjecedt to CCK-8 assay after incubation for the (C) various time points, or were treated with adriamycin at the indicated concentrations and subjected to (D) CCK-8 assay, or were reseeded in 24 -well plates, treated with $5 \mu \mathrm{g} / \mathrm{ml}$ adriamycin for $48 \mathrm{~h}$ and subjected to (E and F) Annexin V-FITC Apoptosis Detection. Values present the means $\pm \mathrm{SD}(\mathrm{n}=3)$. ${ }^{*} \mathrm{P}<0.05$ and ${ }^{* *} \mathrm{P}<0.01$.

with the reporter gene vector carrying a wild-type BCL-2 promoter $(\mathrm{P}<0.05$, vs. the group transfected with the report vector alone) and silencing AP-2 $\alpha$ decreased the luciferase activity $(\mathrm{P}<0.05$, vs. the group transfected with report vector alone). The overexpression or silencing of AP-2 $\alpha$ did not have any obvious effects on luciferase activity in the groups transfected with the reporter gene vector carrying a mutant type BCL-2 promoter (Fig. 4). Therefore, a binding site of AP- $2 \alpha$ in BCL-2 promoter exists through which AP-2 $\alpha$ may regulate gene transcription.

Analysis on the miRNA135a-5p-AP-2 $\alpha-B C L 2$ pathway in apoptosis resistance regulation in gastric cells. The results of mRNA content detection showed that the increase or decrease of miRNA135a-5p inhibited or increased transcription of the $B C L-2$ gene $(\mathrm{P}<0.05$, vs. the non-transfected group), respectively. AP-2 $\alpha$ overexpression increased the BCL-2 mRNA level, while silencing AP-2 $\alpha$ decreased this level directly. In addition, changes in miRNA135a-5p had no significant effect on BCL-2 mRNA in the cells of overexpressed or silenced
AP-2 $\alpha$. The results of protein measurement were concordant with teh mRNA results (Fig. 5).

\section{Discussion}

miRNAs are a wide range of molecules regulating a mass of genes, accounting for $2-3 \%$ of genes in the human genome. In terms of structure, miRNAs are a class of short (18-24 nt) single-stranded non-coding regulatory RNAs that negatively regulate gene expression through complementary to target sites in the 3'-UTR of target mRNAs at the post-transcriptional level, resulting in mRNA degradation or translational repression and consequently control such biological behaviors as tumor genesis and development. Recent studies have demonstrated changes of miRNA expression in a variety of human tumors, which may be a common characteristic of cancer, indicating that miRNAs play critical roles in tumorigenesis. For instance, miR-125b-1, located on a fragile site on chromosome 11q24, has been shown to be associated with breast, lung, ovarian and uterine cancer and play a tumor suppressor role (28). The downregulated 


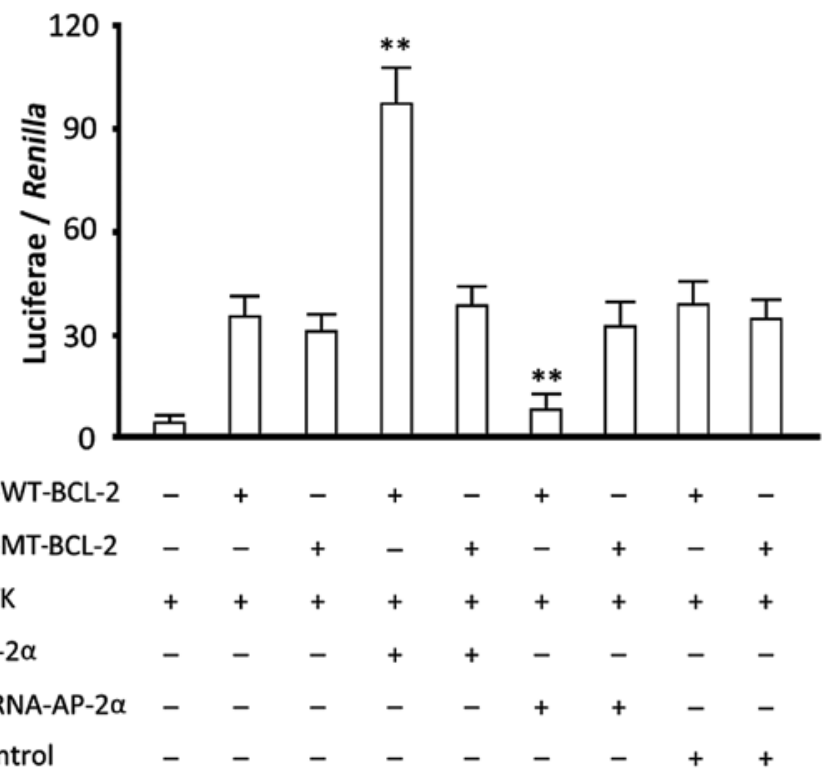

Figure 4. Verification of the binding site of AP- $2 \alpha$ in BCL-2 promoter by the luciferase reporter system. A total of 293 cells were infected with Lv-AP-2 $\alpha$ or Lv-shRNA-AP-2 $\alpha$, reseeded and then transfected with pGL3-WT-BCL2 or pGL3-MT-BCL2 and subjected to luciferase activity assay $48 \mathrm{~h}$ after transfection. The data are presented as the means $\pm \mathrm{SD}(\mathrm{n}=3){ }^{*}{ }^{* *} \mathrm{P}<0.01$.

A

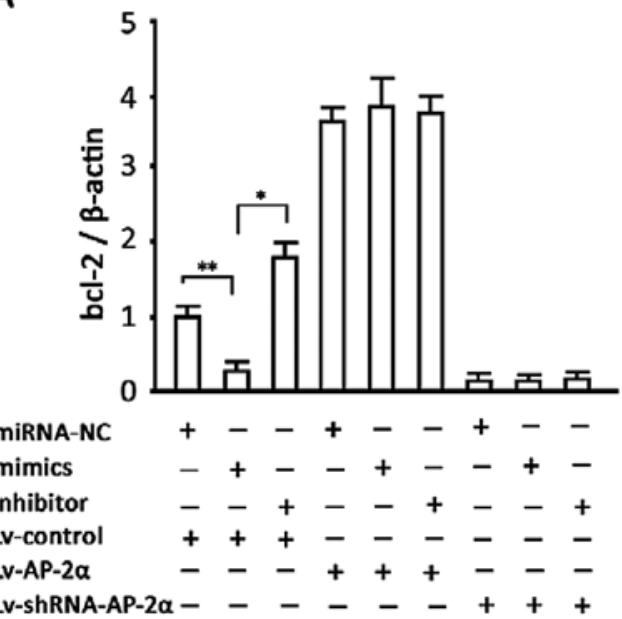

B

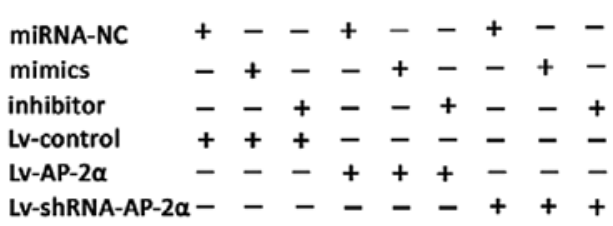

bcl-2

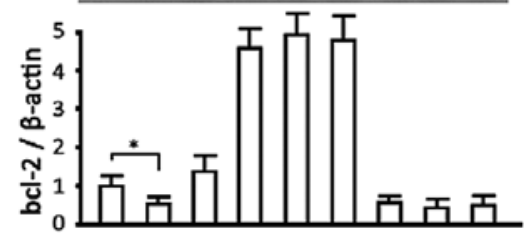

Figure 5. Regulation of BCL-2 by miRNA135a -5p through AP-2 $\alpha$. BGC-823 cells were infected with LV-AP-2 $\alpha$ or LV-shRNA-AP-2 $\alpha$, reseeded and transfected with miRNA135a-5p-mimic or miRNA135a-5p-inhibitor or miRNA-NC and then subject to quantitative PCR detection of BCL-2 mRNA or western blotting of Bcl-2 protein (A and B) $48 \mathrm{~h}$ after transfection. The data are presented as the means $\pm \mathrm{SD}(\mathrm{n}=3)$. ${ }^{*} \mathrm{P}<0.05$ and ${ }^{* *} \mathrm{P}<0.01$.

expression of miR-15a/miR-16-1 is considered one of the main causes of leukemia, lymphoma and prostate cancer (29). miR-143/miR-145 expression is significantly decreased in breast, prostate, and uterine cancer, and lymphoma (30). Let-7 is considered to be a tumor-suppressor gene associated with lung cancer (31). miR-21 is highly expressed in glioblastoma and breast cancer and gene interference experiments have shown it has a tumor-promoting function (32). Similarly, miR-155 expression is increased in Burkitt's and Hodgkin's lymphoma, thus it may also have a cancer-promoting effect (33).

Chemotherapy is the mainstay for the treatment of malignant tumor, however, it is restricted by primary and acquired drug resistance. The mutation of key genes in many drug resis- tance pathways at the genetic or epigenetic level can lead to the occurrence of drug resistance in tumor cells. As a possible regulating way of these key genes, miRNAs are involved in the regulatory function of tumor sensitivity to chemotherapeutic drugs $(34,35)$. Abnormal miRNA expression exists in the majority of cancers (36) and more than half of miRNAs are located on chromosome regions prone to change in tumors. Up- or downregulation of miRNA expression directly results in the abnormal expression of target genes and alters the drug sensitivity of tumor cells by possible signaling pathways. It has been reported that miR-21 overexpression exists in patients with bile duct cancer, thus the patients are not sensitive to gemcitabine, and reducing miR-21 with antisense 
oligodeoxynucleotides enhanced the sensitivity of MCF7 cells to topotecan and promoted apoptosis, which may be mediated by a low expression of BCL2 $(37,38)$. Studies have revealed that drug sensitivity-related miRNAs, also include let-7a, miR-130a, miR-214, mir-27a, miR-451, miR-221/222, miR-199, miR-15b/16, miR-34 and miR-328, most of which are associated with apoptosis (39). Let-7a inhibits apoptosis by downregulating CASP3, an important enzyme in apoptosis and the overexpression of let-7a in A431 and HepG2 cells increased their resistance to adriamycin, paclitaxel and interferon- $\gamma$, while inhibiting let-7a increased chemotherapyinduced apoptosis (40). miR-214 can downregulate PTEN, leading to excessive activation of Akt pathway and cell proliferation, thus patients with miR-214 overexpression did not exhibit sensitivity to cisplatin (41). Low expression of miR-15b and miR-16 in a multidrug resistant gastric cancer cell line, SGC-7901/VCR, maintains its target gene $B C L-2$ at a high level, contributing to the cell resistance to drugs (23). In these studies, BCL-2, a crucial molecule inhibiting apoptosis, is overexpressed in a variety of tumors, resulting in multi-drug resistance. Therefore, examining the relationship between BCL-2 and miRNAs is crucial.

In previous experiments, we screened miRNAs of differential expression in gastric cancer tissue samples and found that miRNA135a-5p may be one of those expressing significant difference (data not shown), so we detected the miR135a-5p expression by quantitative real-time PCR in gastric cancer tissues in this study and found, compared to adjacent tissues, its content in tumor tissues was significantly increased. Subsequently, through a series of experiments, we demonstrated that AP- $2 \alpha$ is one of its target genes, which plays a role in tumor suppression as reported in recent studies $(42,43)$. We tried to knock down miRNA135a-5p to inhibit cell proliferation by increasing AP- $2 \alpha$ levels. However, although AP-2 $\alpha$ was increased, proliferation of BGC-823 cells was not altered substantially. Notably, we treated cells with adriamycin following genetic intervention and found that changes in miRNA135a-5p content affected apoptosis in the tumor cells. This finding suggested that there is a connection between miRNA135a-5p and tumor drug sensitivity. We excluded the direct link by bioinformatics analysis and speculated that miRNA135a-5p may affect crucial drug-sensitive genes through its target gene $A P-2 \alpha$. Since AP- $2 \alpha$ is a transcription factor, we analyzed the genes regulated by it and found that there is a possible binding site in the promoter region of BCL-2 gene, which was confirmed by the luciferase reporter method. To verify the miRNA135a-5p-AP- $2 \alpha$-BCL2 pathway, we altered miRNA135a-5p content in the cells of AP- $2 \alpha$ overexpression and knockdown to observe BCL-2 mRNA and protein contents. The result also confirmed that miRNA135a-5p affects BCL-2 gene expression mediated by AP- $2 \alpha$.

\section{Acknowledgements}

This study was supported by the National Science Foundation of China (81372293 and 81241088 to Y.K.F., 81273161 to K.J.F.), New Century Excellent Talents in Heilongjiang Province University (to Y.K.F.), Post-Doctoral Science Foundation of China (2012M520762 to Y.K.F.), Department of Education of Heilongjiang Province of China (12531736 to Y.M.P.), Program for Innovation Research Team in Science and Technology in Heilongjiang Province University (to Y.K.F. and Z.S.J.) and Program from China Scholarship Council (to Y.K.F.)

\section{References}

1. Villanueva MT: Combination therapy: update on gastric cancer in East Asia. Nat Rev Clin Oncol 8: 690, 2011.

2. Leung WK, Wu MS, Kakugawa Y, et al: Screening for gastric cancer in Asia: current evidence and practice. Lancet Oncol 9: 279-287, 2008

3. Widschwendter M and Jones PA: DNA methylation and breast carcinogenesis. Oncogene 21: 5462-5482, 2002.

4. Schoof CR, Botelho EL, Izzotti A and Vasques Ldos R: MicroRNAs in cancer treatment and prognosis. Am J Cancer Res 2: 414-433, 2012.

5. Hu J, Cheng Y, Li Y, et al: microRNA-128 plays a critical role in human non-small cell lung cancer tumourigenesis, angiogenesis and lymphangiogenesis by directly targeting vascular endothelial growth factor-C. Eur J Cancer 50: 2336-2350, 2014.

6. Nakata K, Ohuchida K, Mizumoto K, et al: MicroRNA-10b is overexpressed in pancreatic cancer, promotes its invasiveness, and correlates with a poor prognosis. Surgery 150: 916-922, 2011.

7. Nishida N, Yamashita S, Mimori K, et al: MicroRNA-10b is a prognostic indicator in colorectal cancer and confers resistance to the chemotherapeutic agent 5-fluorouracil in colorectal cancer cells. Ann Surg Oncol 19: 3065-3071, 2012.

8. Liu K, Li G, Fan C, Zhou X, Wu B and Li J: Increased expression of microRNA-21and its association with chemotherapeutic response in human colorectal cancer. J Int Med Res 39: 2288-2295, 2011.

9. Jiang L, Mao P, Song L, et al: miR-182 as a prognostic marker for glioma progression and patient survival. Am J Pathol 177: 29-38, 2010.

10. Adachi R, Horiuchi S, Sakurazawa Y, Hasegawa T, Sato K and Sakamaki T: ErbB2 down-regulates microRNA-205 in breast cancer. Biochem Biophys Res Commun 411: 804-808, 2011.

11. Chao A, Lin CY, Lee YS, et al: Regulation of ovarian cancer progression by microRNA-187 through targeting Disabled homolog-2. Oncogene 31: 764-775, 2012.

12. Chai H, Liu M, Tian R, Li X and Tang H: miR-20a targets BNIP2 and contributes chemotherapeutic resistance in colorectal adenocarcinoma SW480 and SW620 cell lines. Acta Biochim Biophys Sin (Shanghai) 43: 217-225, 2011.

13. Gocek E, Wang X, Liu X, Liu CG and Studzinski GP: MicroRNA-32 upregulation by 1,25-dihydroxyvitamin D3 in human myeloid leukemia cells leads to Bim targeting and inhibition of AraC-induced apoptosis. Cancer Res 71: 6230-6239, 2011.

14. Kovalchuk O, Filkowski J, Meservy J, et al: Involvement of microRNA-451 in resistance of the MCF-7 breast cancer cells to chemotherapeutic drug doxorubicin. Mol Cancer Ther 7: 2152-2159, 2008.

15. Chen J, Tian W, Cai H, He H and Deng Y: Down-regulation of microRNA-200c is associated with drug resistance in human breast cancer. Med Oncol 29: 2527-2534, 2012.

16. Zhang Z, Li Z, Gao C, et al: miR-21 plays a pivotal role in gastric cancer pathogenesis and progression. Lab Invest 88: 1358-1366, 2008.

17. Chan SH, Wu CW, Li AF, Chi CW and Lin WC: miR-21 microRNA expression in human gastric carcinomas and its clinical association. Anticancer Res 28: 907-911, 2008.

18. Lai KW, Koh KX, Loh M, et al: MicroRNA-130b regulates the tumour suppressor RUNX3 in gastric cancer. Eur J Cancer 46: 1456-1463, 2010.

19. Zhang X, Zhu W, Zhang J, et al: MicroRNA-650 targets ING4 to promote gastric cancer tumorigenicity. Biochem Biophys Res Commun 395: 275-280, 2010.

20. Wu Q, Jin H, Yang Z, et al: MiR-150 promotes gastric cancer proliferation by negatively regulating the pro-apoptotic gene EGR2. Biochem Biophys Res Commun 392: 340-345, 2010.

21. Jiang Z, Guo J, Xiao B, et al: Increased expression of miR-421 in human gastric carcinoma and its clinical association. $J$ Gastroenterol 45: 17-23, 2010.

22. Xiao B, Guo J, Miao Y, et al: Detection of miR-106a in gastric carcinoma and its clinical significance. Clin Chim Acta 400: 97-102, 2009 
23. Xia L, Zhang D, Du R, et al: miR-15b and miR-16 modulate multidrug resistance by targeting BCL2 in human gastric cancer cells. Int J Cancer 123: 372-379, 2008.

24. Zhu W, Zhu D, Lu S, et al: miR-497 modulates multidrug resistance of human cancer cell lines by targeting BCL2. Med Oncol 29: 384-391, 2012.

25. Orso F, Penna E, Cimino D, et al: AP-2alpha and AP-2gamma regulate tumor progression via specific genetic programs. FASEB J 22: 2702-2714, 2008

26. Jonckheere N, Fauquette V, Stechly L, et al: Tumour growth and resistance to gemcitabine of pancreatic cancer cells are decreased by AP-2alpha overexpression. Br J Cancer 101: 637-644, 2009

27. Allouche A, Nolens G, Tancredi A, et al: The combined immunodetection of AP-2alpha and YY1 transcription factors is associated with ERBB2 gene overexpression in primary breast tumors. Breast Cancer Res 10: R9, 2008.

28. Thorsen J, Aamot HV, Roberto R, Tjonnfjord GE, Micci F and Heim S: Myelodysplastic syndrome with a $t(2 ; 11)(\mathrm{p} 21 ; \mathrm{q} 23-24)$ and translocation breakpoint close to miR-125b-1. Cancer Genet 205: 528-532, 2012.

29. Liu J, Chen G, Feng L, et al: Loss of p53 and altered miR15-a/16-1 $\rightarrow$ MCL-1 pathway in CLL: insights from TCL1$\mathrm{Tg}$ :p53(-/-) mouse model and primary human leukemia cells Leukemia 28: 118-128, 2014.

30. Kent OA, Chivukula RR, Mullendore M, et al: Repression of the miR-143/145 cluster by oncogenic Ras initiates a tumorpromoting feed-forward pathway. Genes Dev 24: 2754-2759, 2010.

31. Hertel J, Bartschat S, Wintsche A, Otto C and Stadler PF: Evolution of the let-7 microRNA family. RNA Biol 9: 231-241, 2012.

32. Wickramasinghe NS, Manavalan TT, Dougherty SM, Riggs KA, $\mathrm{Li} \mathrm{Y}$ and Klinge CM: Estradiol downregulates miR-21 expression and increases miR-21 target gene expression in MCF-7 breast cancer cells. Nucleic Acids Res 37: 2584-2595, 2009.
33. Jones K, Nourse JP, Keane C, Bhatnagar A and Gandhi MK Plasma microRNA are disease response biomarkers in classical Hodgkin lymphoma. Clin Cancer Res 20: 253-264, 2014.

34. Blower PE, Verducci JS, Lin S, et al: MicroRNA expression profiles for the NCI-60 cancer cell panel. Mol Cancer Ther 6: 1483-1491, 2007.

35. Salter KH, Acharya CR, Walters KS, et al: An integrated approach to the prediction of chemotherapeutic response in patients with breast cancer. PLoS One 3: e1908, 2008.

36. Bertino JR, Banerjee D and Mishra PJ: Pharmacogenomics of microRNA: a miRSNP towards individualized therapy. Pharmacogenomics 8: 1625-1627, 2007.

37. Meng F, Henson R, Lang M, et al: Involvement of human micro-RNA in growth and response to chemotherapy in human cholangiocarcinoma cell lines. Gastroenterology 130: 2113-2129, 2006.

38. Si ML, Zhu S, Wu H, Lu Z, Wu F and Mo YY: miR-21-mediated tumor growth. Oncogene 26: 2799-2803, 2007.

39. Bussing I, Slack FJ and Grosshans H: let-7 microRNAs in development, stem cells and cancer. Trends Mol Med 14: 400-409, 2008.

40. Tsang WP and Kwok TT: Let-7a microRNA suppresses therapeutics-induced cancer cell death by targeting caspase-3. Apoptosis 13: 1215-1222, 2008

41. Yang H, Kong W, He L, et al: MicroRNA expression profiling in human ovarian cancer: miR-214 induces cell survival and cisplatin resistance by targeting PTEN. Cancer Res 68: 425-433, 2008.

42. Xu M, Chen X, Chen N, et al: Synergistic silencing by promoter methylation and reduced AP-2 $\alpha$ transactivation of the proapoptotic HRK gene confers apoptosis resistance and enhanced tumor growth. Am J Pathol 182: 84-95, 2013.

43. Fu L, Chen W, Guo W, et al: Berberine Targets AP-2/hTERT, NF- $\kappa \mathrm{B} / \mathrm{COX}-2$, HIF-1 $\alpha / \mathrm{VEGF}$ and Cytochrome-c/Caspase Signaling to Suppress Human Cancer Cell Growth. PLoS One 8: e69240, 2013 